\title{
Research Article \\ Improving Volatility Risk Forecasting Accuracy in Industry Sector
}

\author{
S. Al Wadi \\ Department of Risk Management and Insurance, Faculty of Management and Finance, The University of Jordan, Amman, Jordan \\ Correspondence should be addressed to S. Al Wadi; sadam_alwadi@yahoo.co.uk
}

Received 5 August 2017; Revised 6 October 2017; Accepted 10 October 2017; Published 7 November 2017

Academic Editor: Niansheng Tang

Copyright (C) 2017 S. Al Wadi. This is an open access article distributed under the Creative Commons Attribution License, which permits unrestricted use, distribution, and reproduction in any medium, provided the original work is properly cited.

\begin{abstract}
Recently, the volatility of financial markets has contributed a necessary part to risk management. Volatility risk is characterized as the standard deviation of the constantly compound return per day. This paper presents forecasting of volatility for the Jordanian industry sector after the crisis in 2009. ARIMA and ARIMA-Wavelet Transform (WT) have been conducted in order to select the best forecasting model in the content of industry stock market data collected from Amman Stock Exchange (ASE). As a result, the researcher found that ARIMA-WT has more accuracy than ARIMA directly. The results have been introduced using MATLAB 2010a and R programming.
\end{abstract}

\section{Introduction}

Recently, several financial categories of research have been concerned with the forecasting volatility modeling since it plays an important role in risk management and financial asset pricing such as bonds and stocks. The forecasting accuracy helps financial market participants to estimate future risks. After that the regulators can make decisions regarding the financial instruments (Bollersleva et al. 2014).

The experience of market risk was formally predicted with the accurate volatility forecast. The high fluctuation of stock prices highlights the importance of volatility forecast. Recently, a list of volatility models has been suggested in the educational works of literature for testing the fundamental trade-off between risks and return of financial assets, and for investigating the causes and consequences of the volatility dynamic in the economy [1].

One of the famous volatility models is GARCH (Generalized Autoregressive Conditional Heteroskedasticity) model which is commonly used for estimating and forecasting financial market volatility. Based on Engle's ARCH (Autoregressive Conditional Heteroskedasticity) in 1982 and Bolllerselv's GARCH models in 1986, plenty of GARCH models, such as NAGARCH, GJR-GARCH, FIGARCH, and EGARCH, are created in options markets, exchange, and bonds. A huge percentage of studies focused on the stock market volatility.
Trück and Liang examined the performance of different models (GARCH, TARCH, TGARCH, and ARMA) in 2012 [2]. Researchers found that the TARCH models provided the best results in the gold market.

In this study, the volatility of the stock market prices will be modeled using WT. Also, this article improves the forecasting accuracy in the content of volatility model using industry data from ASE by combining ARIMA model with WT; then finally the results of ARIMA model directly are compared with WT + ARIMA. Generally, the volatility should be based on ARIMA model which has great concentration in finance and economic fields. George Box and Gwilym Jenkins (ARIMA) is a forecasting model which was popularized in the 1970s. ARIMA model is defined as: $\operatorname{ARIMA}(p, d, q)$ where: $P$ : order of autoregressive part (AR), $d$ : degree of first differentiation (I), and $q$ : order of the first moving part (MA). According to that, ARIMA models create the best forecast for the time series. This method is not costly compared to other methods in the quantitative model [3]. The comparison tested the accuracy of these models by using RMSE and MAPE.

This paper is organized as follows: Section 2 provides a literature review; Section 3 provides data description; Section 4 reports the results from an empirical application of the stock market; Section 5 concludes. 


\section{Literature Review}

2.1. Volatility Model. Recently, forecasting the financial data got high attention such as [4-7]. More specifically, forecasting volatility is a well-liked research agenda in stock market data. There are an increasing number of models for forecasting volatility fields. Reference [8] studies ARCH, GARCH, EGARCH, and exponential GARCH for stock market data with sample size 2200. Bentes [9] studies the adequacy of GARCH-class models in describing the gold volatility behavior and compare the out-of-sample predictive ability models (GARCH, IGARCH and FIGARCH) based on three evaluation criterions: mean absolute error (MAE), root mean squared error (RMSE), and Theil's inequality coefficient (TIC) over a long time frame of over 39 years in order to provide a general picture of the overall behavior of the time series. Reference [10] used ARCH and GARCH methods to determine the persistency of volatilities. Reference [11] explored the effect of including the volatility index (VIX) as a benchmark of expected short-term market (options and futures) and trading volume (VO) within the volatility forecasting model. Reference [12] examines the improvement of accuracy in forecasting using a hybrid model as opposed to traditional GARCH models. Reference [13] studies forecasting the volatility of Tehran Stock Exchange (TSE) and they concentrated on the empirical estimates of ARMA, singleregime GARCH, and MRS-GARCH models, together with the in-the-sample and the out-of-sample forecast evaluation. Reference [14] examines and predicts aggregate volatility, and the researcher developed a model of individual returns to the study of volatility. Reference [15] examined a new class of volatility forecasting models and they noticed significant improvements in the accuracy of the resulting forecasts compared to the forecasts from some of the most popular existing models. They found that the HARQ model is slightly more subtle while the HAR places greater weight on the weekly and monthly lags.

In the WT field, there are some results in the forecasting accuracy in the stock market data that have been introduced such as [16]. Reference [17] used financial data from ASE to test three methods (box plot, $Z$-score, and Wavelet Transform Asymmetric Winsorized Mean (WTAWM)) for outlier detection. Reference [18] introduced Multifractal analysis that is provided using the so-called Wavelet Transform Modulus Maxima approach which is beneficial for the forecasting and the simulations of most European and Asian stock indexes. Reference [19] predicts stock market index of Tehran Stock Exchange by combining of ARIMA, neural network, and WT in order to predict trend of the market. Moreover, after intensive research in the literature, the researcher has not found any research that combines WT with ARIMA model in modeling and forecasting volatility of industry time series data in the content of ASE.

\section{Mathematical Formulations}

The definition of volatility, WT, and ARIMA models will be discussed. After that, the several approaches to accuracy will be listed.
3.1. Volatility Formula. In risk management area, volatility is defined as the standard deviation of the continuously compounded return per day. Define $S_{i}$ as the close price at the end of day $i$. The continuously compounded return per day for the close price on day $i$ as

$$
R=\ln \frac{S_{i}}{S_{i-1}} .
$$

A variable's volatility, $\sigma_{t}$, is defined as the standard deviation of $R_{i}$ 's at time $t$.

$$
\sigma_{t}=\sqrt{\frac{\sum_{i=1}^{n}\left(R_{i}-\bar{R}\right)^{2}}{n-1}},
$$

where $\bar{R}$ is the arithmetic mean of $R_{i}$ 's $[1,20]$.

3.2. Wavelet Transform Equation. WT is defined by $[1,21,22]$ as

$$
\psi_{j, k}(t)=2^{j / 2} \psi\left(2^{j} t-k\right), \quad j, k \in Z ; z=\{0,1,2, \ldots\}
$$

where $\psi$ is defined as a real-valued function having compactly supported, and $\int_{-\infty}^{\infty} \psi(t) d t=0$.

Generally, WT were calculated by using dilation equations and were defined as

$$
\begin{aligned}
& \phi(t)=\sqrt{2} \sum_{k} l_{k} \phi(2 t-k), \\
& \psi(t)=\sqrt{2} \sum_{k} h_{k} \phi(2 t-k),
\end{aligned}
$$

where $\phi(2 t-k)$ shows the father wavelet, and $\psi(t)$ represents the mother wavelet. Father wavelet introduces the high scale approximation components of the signal, while the mother wavelet introduces the deviations from the approximation components. The father wavelet produces the scaling coefficients, while mother wavelet estimates the differencing coefficients. Father wavelet defines high pass filters coefficients $\left(l_{k}\right)$ and the lower pass filter coefficients $\left(h_{k}\right)$ are defined, respectively, as follows $[1,22]$ :

$$
\begin{aligned}
& l_{k}=\sqrt{2} \int_{-\infty}^{\infty} \phi(t) \phi(2 t-k) d t \\
& h_{k}=\sqrt{2} \int_{-\infty}^{\infty} \psi(t) \psi(2 t-k) d t .
\end{aligned}
$$

HWT is the oldest and simplest example in the wavelet transforms and it can be defined as

$$
\psi^{H}(t)= \begin{cases}1, & 0 \leq t \leq \frac{1}{2} \\ -1, & \frac{1}{2} \leq t \leq 1 \\ 0, & \text { Otherwise. }\end{cases}
$$


For the HWT:

$$
\begin{array}{r}
l_{k}=\sqrt{2} \int_{-\infty}^{\infty} \phi(t) \phi(2 t-k) d t= \begin{cases}1, & 0 \leq t \leq 1 \\
0, & \text { otherwise }\end{cases} \\
\quad \text { for } N=2, l_{k}=\left\{\frac{1}{\sqrt{2}}, \frac{1}{\sqrt{2}}\right\}, h_{k}=\left\{\frac{1}{\sqrt{2}}, \frac{-1}{\sqrt{2}}\right\} .
\end{array}
$$

The mother wavelet satisfies the following two conditions:

$$
\begin{array}{r}
\int_{-\infty}^{\infty} \psi(t) d t=0, \\
\int_{-\infty}^{\infty}|\psi(t)|<\infty, \\
\int_{-\infty}^{\infty} \frac{\left|\psi_{1}(\omega)\right|^{2}}{|\omega|} d \omega<\infty,
\end{array}
$$

where $\psi_{1}(\omega)$ presents WT.

The HWT was improved and developed the frequency-domain characteristics by Daubechies WT (DWT). However, there is no specific formula for DWT. Therefore, the square gain function of their scaling filter is used; it is defined as (Gencay et al., [22]):

$$
g(f)=2 \cos ^{l}(\pi f) \sum_{l=0}^{l / 2-1}\left(\begin{array}{c}
\frac{l}{2}-1+l \\
l
\end{array}\right) \sin ^{2 l}(\pi f),
$$

where $l$ is positive number and represents the length of the filter. For more details and examples, see $[1,22,23]$.

\subsection{Autoregressive Integrated Moving-Average Model} $(A R I M A(p, d, q))$. The auto-regressive moving average (ARMA) models are used in stock market to illustrate stationary time series. The ARMA model is a combination of an autoregressive (AR) model and a moving average (MA) model. A time series $\left\{e_{t}\right\}$ is said to be a white noise (WN) process, $\left\{Y_{t}\right\}$ is called Gaussian process iff for all $t, e_{t}$ is iid $N\left(0, \sigma^{2}\right)[1]$. if

A time series $\left\{Y_{t}\right\}$ is said to follow the $\operatorname{ARMA}(p, q)$ model

$$
\begin{aligned}
Y_{t}= & i+\phi_{1} Y_{t-1}+\phi_{2} Y_{t-2}+\cdots+\phi_{p} Y_{t-p}+e_{t}-\dot{e}_{1} e_{t-1} \\
& -\dot{e}_{2} e_{t-2}-\cdots-\dot{e}_{q} e_{t-q}
\end{aligned}
$$

where $p$ and $q$ are non-negative integers, $p$ represents order of autoregressive part (AR), $q$ is defined as order of the first moving part (MA) and $\left\{e_{t}\right\}$ is the white noise (WN) process.

An extension of the ordinary ARMA model is the autoregressive integrated moving-average model $(\operatorname{ARIMA}(p$, $d, q)$ ) given by

$$
\phi_{p}(B)(1-B)^{d} Y_{t}=\grave{\mathrm{e}}_{0}+\grave{\mathrm{e}}_{q}(B) e_{t},
$$

where $p, d$ and $q$ denote orders of auto-regression, integration (differencing) and moving average, respectively.

When $d=0$, the ARIMA model reduces to the ordinary ARMA model.
ARIMA model is the majority famous way of forecasting since there is no need for any assumptions and it is not limited to specific type of pattern. These models can be fitted to any set of time series data (stationary or non-stationary) by estimating the parameters $p, d$, and $q$ to be suitable with the required dataset.

3.4. Accuracy Criteria Equation. The author used some criteria in order to make fair comparison between ARIMA and ARIMA-WT that can be presented in this section. Some types of accuracy criteria have used root means squared error (RMSE), percentage root mean absolute percentage error (MAPE), and mean absolute error (MAE). For the mathematical formulas, refer to [24].

\section{Methodology and Results}

In order to show forecasting volatility risk for the industry sector in the stock market, daily close price is used from industry after crises 2009 for the time period 2009-2015 selected from ASE.

4.1. Comparison with ARIMA and ARIMA-WT and Accuracy Criteria. The DWT (Discrete wavelet transform) converts the data into two sets: approximation series (CA1 $(n))$ and details series (DA1 $(n))$. These two series presented good behavior for the data set, especially with the industry data, since it is significantly fluctuated. Then, the transformed data can be predicted more accurately. The reason for the good behavior of these two series is the filtering effect of the dWT (Daubechies WT); dWT is used in this article since it is the best WT function. Therefore, the methodology of the comparing in this study is described as follows.

Firstly, decompose through dWT the available historical return data.

Secondly, develop the fitted ARIMA model directly.

Thirdly, use specific ARIMA model fitted to each one of the approximation series to make the forecasting, which means make forecasting using dWT with ARIMA model.

Finally, the results in the second and third points are compared.

Figure 1 summarizes the methodology of this article.

Referring to Figure 1, then notice the following:

(1) ARIMA model has been used since it is suitable with linear and stationary data, and WT is a suitable model for nonstationary and nonlinear data. Therefore, the combination between ARIMA and WT will give a strong model to improve the forecasting accuracy.

(2) In this article, the decomposition for level 4 has been used. However, the author can use any other level since the smoothed data only is used forecasting accuracy. Therefore, the level of decomposition is unjustified.

(3) dWT (Daubechies WT) is used in this article since it is well known that dWT is the best function in the WT field. 


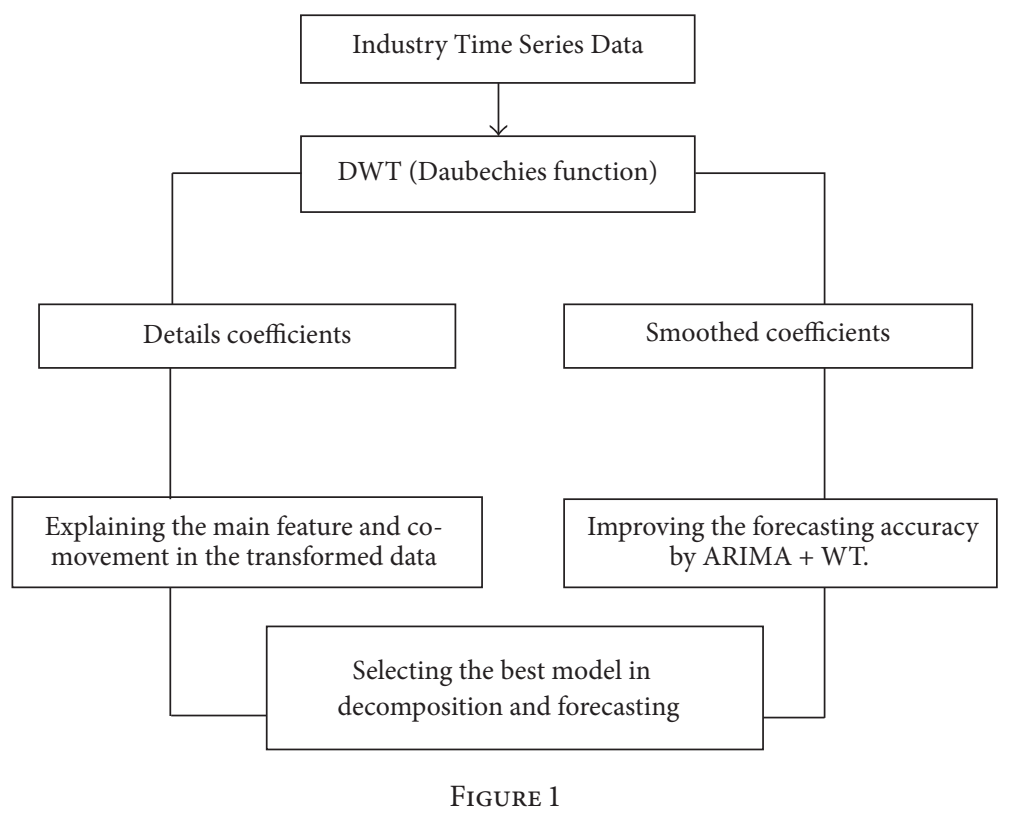

4.2. Daily Forecasting Volatility of ARIMA-WT. The forecasting volatility of ARIMA-WT is presented in Figure 2 for each Jordanian industry sector using dWT. The forecasting volatility of ARIMA-WT and ARIMA directly for Jordanian industry data appeared in Table 1 . The percentage of volatility is high in the first two years because of the crisis of 2009. After that, we found that there is a stability of volatility for all banks after the crisis.

Regarding Figure 2, the details coefficients are presented the levels $\left(d_{1}, d_{2}, d_{3}, d_{4}\right.$ and $\left.d_{5}\right)$ which will be used to explain the main fluctuations and real events in the dataset. The approximation coefficients $\left(a_{1}\right)$ consist of the main features of the dataset which will be used in forecasting.

Therefore, $d 4$ will be used in this study to explain the events that occur in this sector. Generally, the growth of this sector slowed over the years 2009-2014 faster than the slowdown of the national economy as a whole. The growth of the manufacturing sector reached $2.3 \%$ as an annual average during the period 2009-2014, compared to the growth of the national economy as a whole averaging about $3 \%$. More specifically, the data was very fluctuated as: firstly (the observations before number 200) it means that, in 2009, these fluctuations were caused by the global financial crisis. The data become more fluctuated in the observations number 800, which means during the years 2012 and 2013 then the data become very stable at the end. Also, in regard to $d_{1}$, there is a high fluctuation in the observations between 400 and 600, which means the year number 2011; some events will be presented that have effect on the data and make the mentioned fluctuations:

(1) Signing a memorandum of understanding between Amman Stock Exchange and the Egyptian Exchange.

(2) ASE has finished the stage of preparing the new website.

(3) New version of the Electronic Trading System.
TABLE 1: The forecasting results.

\begin{tabular}{lcc}
\hline & ARIMA directly & ARIMA + WT \\
\hline RMSE & 1.56 & 0.6 \\
MAPE & 520 & 286 \\
MAE & 1.34 & 0.4 \\
\hline
\end{tabular}

(4) ASE launches the Internet Trading Service.

(5) The meetings of the Working Committee of the Federation of Euro-Asian Stock Exchanges (FEAS).

(6) S\&P Indices and Arab Federation of Exchanges create S\&P AFE 40 Index.

(7) ASE receives an economic delegation from the French Embassy.

(8) ASE receives a delegation from European Bank For Reconstruction and Development.

(9) The national economy was able to achieve a real growth in GDP during the three first quarters of 2014.

(10) Amman Stock Exchange receives a delegation from Libyan Stock Market.

Regarding the second target of this paper, Table 1 will show the results of the forecasting accuracy using ARIMA model directly and ARIMA-WT.

For the sake of fair comparison the same number of data set is selected. The suitable forecasted model for forecasting the sample data is the fitted dWT- $\operatorname{ARIMA}(2,0,2)$ with RSME equal to 0.6 as presented in Table 1 . While the Fitted ARIMA model directly is $\operatorname{ARIMA}(1,0,2)$ with RMSE equal to 1.56 which means that the forecasting accuracy has improved by combining dWT + ARIMA model. 

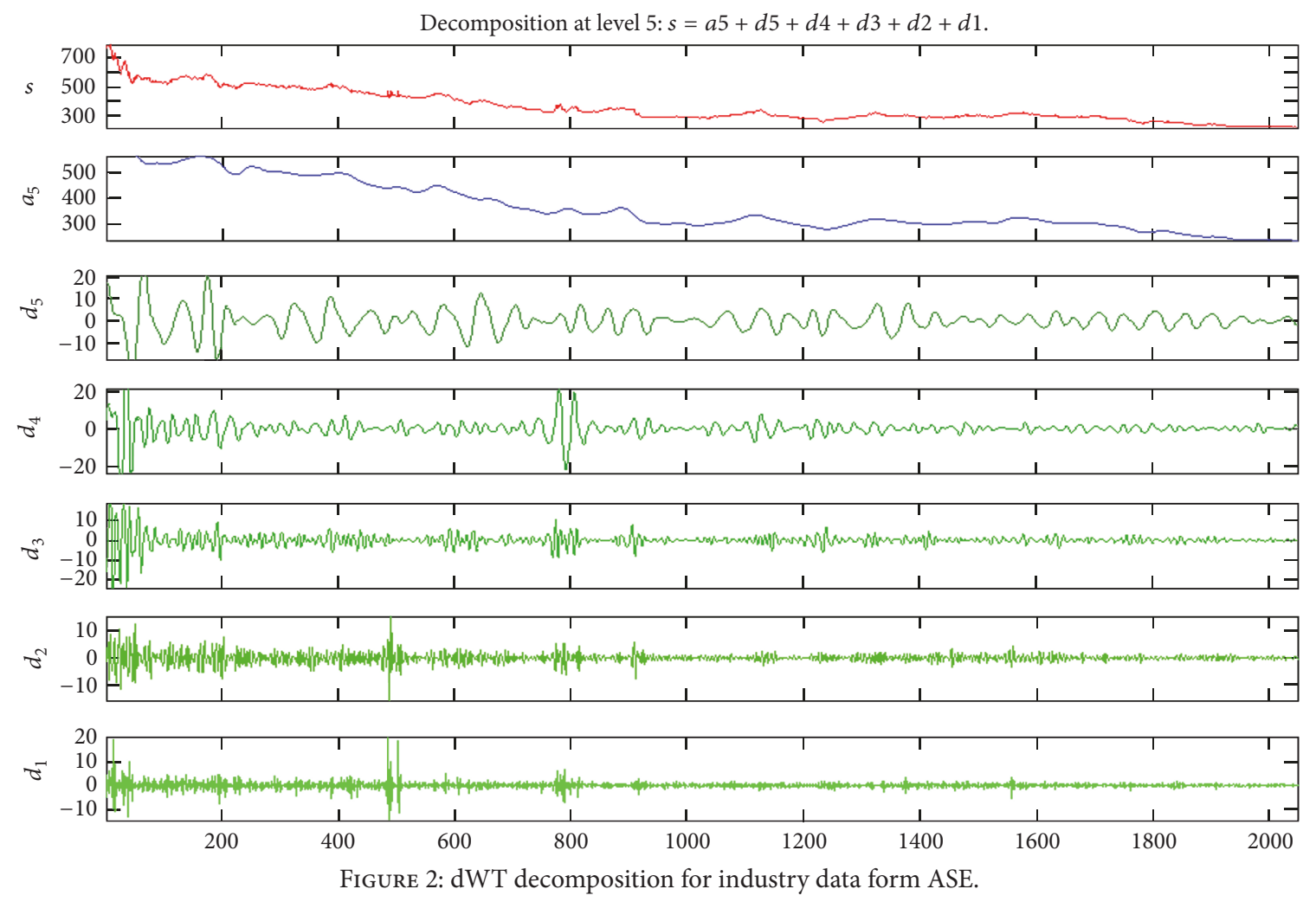

\section{Conclusion}

ARIMA model is the most general way of forecasting since there is no need for any assumptions and it is not limited to a specific type of pattern. These models can be fitted to any set of time series data (stationary or non-stationary) by estimating the parameters $p, d$, and $q$ to be suitable with the required dataset [25]. In this study, firstly, the volatility of the stock price is modeled using wavelet method. Secondly, we compared ARIMA with Wavelet transform with ARIMA model in the content of forecasting accuracy. Thirdly, we tested the accuracy of these models by using RMSE, MAPE, and MSE assessing functions. Finally, the forecasting accuracy has improved using the suggested model. Indeed, we found ARIMA-WT to be a suitable model in the content of industry sector.

\section{Conflicts of Interest}

The authors declare that they have no conflicts of interest.

\section{References}

[1] J. J. Jaber, N. Ismail, S. A. Wadi, and M. H. Saleh, Forecasting of volatility risk for Jordanian banking sector. Far East Journal of Mathematical Sciences, vol. 101, no. 7, pp. 1491-1507, 2017.

[2] S. Trück and K. Liang, "Modelling and forecasting volatility in the gold market," Journal of International Banking and Finance, vol. 9, pp. 48-80, 2012.

[3] A. Pankratz, Forecasting with Univariate Box - Jenkins Models: Concepts and Cases, John Wiley Sons, 1993.
[4] A. Awajan, M. T. Ismail, and S. Al Wadi, "A hybrid EMD-MA for forecasting stock market index," Italian Journal of Pure and Applied Mathematics, vol. 38, no. 1, pp. 1-20, 2017.

[5] A. M. Awajan, M. T. Ismail, and S. Al Wadi, "Forecasting Time Series Data Using EMD-HW Bagging," International Journal of Statistics and Economics, vol. 18, no. 3, pp. 9-21, 2017.

[6] S. R. Albotoush, Al. Wadi, R. Mohammad, and A. Abu Hilal, "Forecasting of Handled Cargo in Aqaba 2030," International Journal of Ecological Economics and Statistics, vol. 38, no. 3, pp. 102-113, 2017.

[7] A. M. Awajan, M. T. Ismail, and S. AL Wadi, "A hybrid approach EMD-MA for short-term forecasting of daily stock market time series data," Journal of Internet Banking and Commerce, vol. 22, no. 1, pp. 1-10, 2017.

[8] L. Gang and L. Yong, "Forecasting copper futures volatility under model uncertainty, Resources Policy," The International Journal of Minerals Policy and Economics, vol. 46, pp. 167-176, 2015.

[9] S. R. Bentes, "Forecasting volatility in gold returns under the GARCH, IGARCH and FIGARCH frameworks: New evidence," Physica A: Statistical Mechanics and its Applications, vol. 438, pp. 355-364, 2015.

[10] A. Joukar and I. Nahmens, "Volatility forecast of construction cost index using general autoregressive conditional heteroskedastic method," Journal of Construction Engineering and Management, vol. 142, no. 1, Article ID 04015051, 2015.

[11] D. S. Kambouroudis and D. G. McMillan, "Does VIX or volume improve GARCH volatility forecasts?" Applied Economics, vol. 48, no. 13, pp. 1210-1228, 2015.

[12] W. Kristjanpoller and M. C. Minutolo, "Gold price volatility: a forecasting approach using the artificial neural networkGARCH model," Expert Systems with Applications, vol. 42, no. 20, pp. 7245-7251, 2015. 
[13] E. Abounoori, Z. Elmi, and Y. Nademi, "Forecasting Tehran stock exchange volatility; Markov switching GARCH approach," Physica A: Statistical Mechanics and its Applications, vol. 445, pp. 264-282, 2016.

[14] S. J. Byun, "The usefulness of cross-sectional dispersion for forecasting aggregate stock price volatility," Journal of Empirical Finance, vol. 36, pp. 162-180, 2016.

[15] T. Bollerslev, A. J. Patton, and R. Quaedvlieg, "Exploiting the errors: A simple approach for improved volatility forecasting," Journal of Econometrics, vol. 192, no. 1, pp. 1-18, 2016.

[16] S. Al Wadi, A. Hamarsheh, and H. Alwadi, "Maximum overlapping discrete wavelet transform in forecasting banking sector," Applied Mathematical Sciences, vol. 7, no. 77-80, pp. 3995-4002, 2013.

[17] A. M. H. Al-Khazaleh, S. Al Wadi, and F. Ababneh, "Wavelet transform asymmetric winsorized mean in detecting outlier values," Far East Journal of Mathematical Sciences, vol. 96, no. 3, pp. 339-351, 2015.

[18] A. Puckovs and A. Matvejevs, "Wavelet Transform Modulus Maxima Approach for World Stock Index Multifractal Analysis," Information Technology and Management Science, vol. 15, no. 1, 2012.

[19] A. Abbasi, M. A. Aghaei, and M. M. Zadehfard, "Case study of price index of Tehran Stock Exchange," ournal Forecasting Stock Market Using Wavelet Transforms and Neural Networks and ARIMA, vol. 5, no. 3, pp. 31-40, 2015.

[20] J. Hull, Risk management and financial institutions, vol. 733, John Wiley Sons, 2012.

[21] C. Chang and P. Moretin, "A wavelet analysis for time series," in Nonparametric Statistics, vol. 10, pp. 1-46, 1998.

[22] R. Gencay, F. Seluk, and B. Whitcher, An Introduction to Wavelets and Other Filtering Methods in Finance and Economics, Academic Press, New York, 2002.

[23] I. Daubechies, Ten Lectures on Wavelets, SIAM, Philadelphia, Pa, USA, 1992.

[24] F. M. Al-Rawashdi, S. Al Wadi, and M. HasanSaleh, "Wavelet methods in forecasting for insurance companies listed in amman stock exchange," European Journal of Economics, Finance and Administrative Sciences, vol. 82, 2015.

[25] A. Mahir and A. Al-Khazaleh, "Estimation of missing data by using the filtering process in a time series modeling," Applied Mathematical Sciences, vol. 3, no. 35, pp. 1715-1726, 2008.

[26] G. Janacek and L. Swift, Time series forecasting, simulation and applications. Ellis Hoe wood limited, simulation and applications. Ellis Hoe wood limited, England, 1993. 


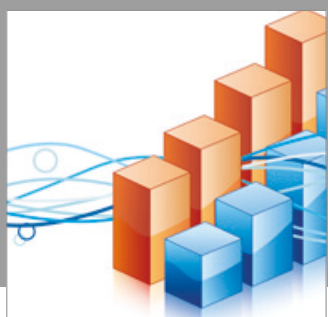

Advances in

Operations Research

vatersals

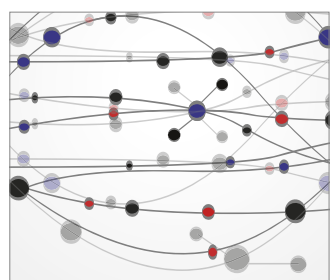

\section{The Scientific} World Journal
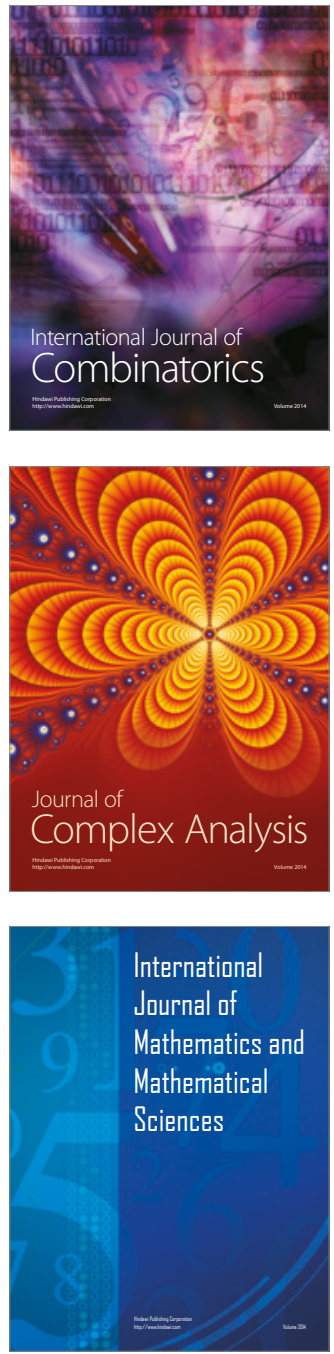
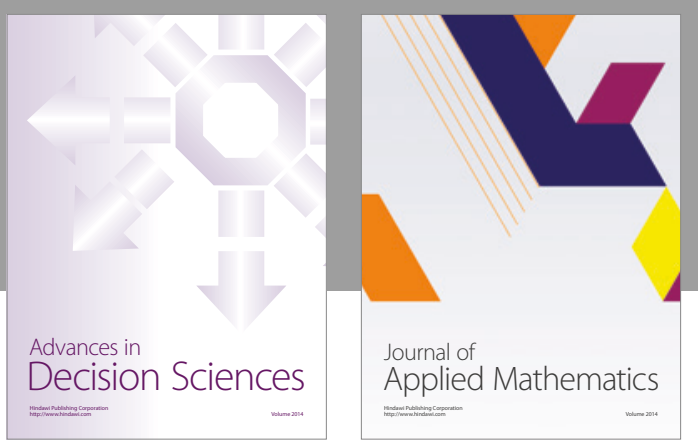

Algebra

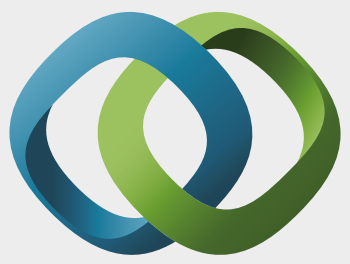

\section{Hindawi}

Submit your manuscripts at

https://www.hindawi.com
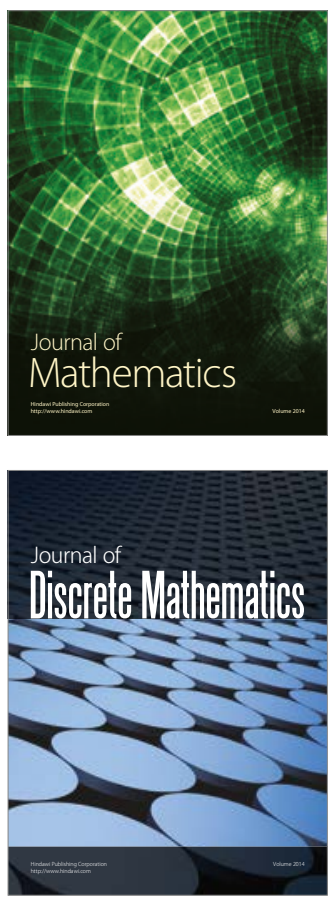

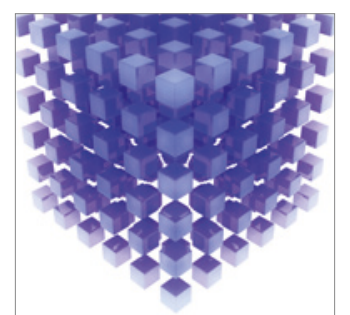

Mathematical Problems in Engineering
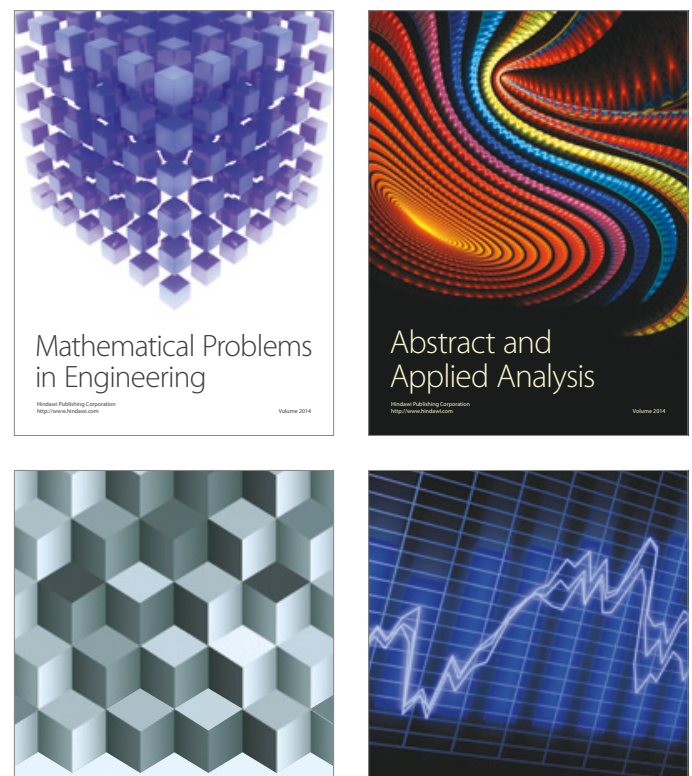

Journal of

Function Spaces

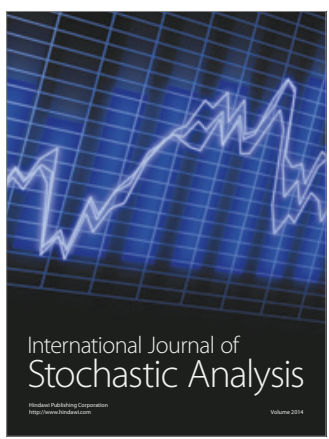

Probability and Statistics
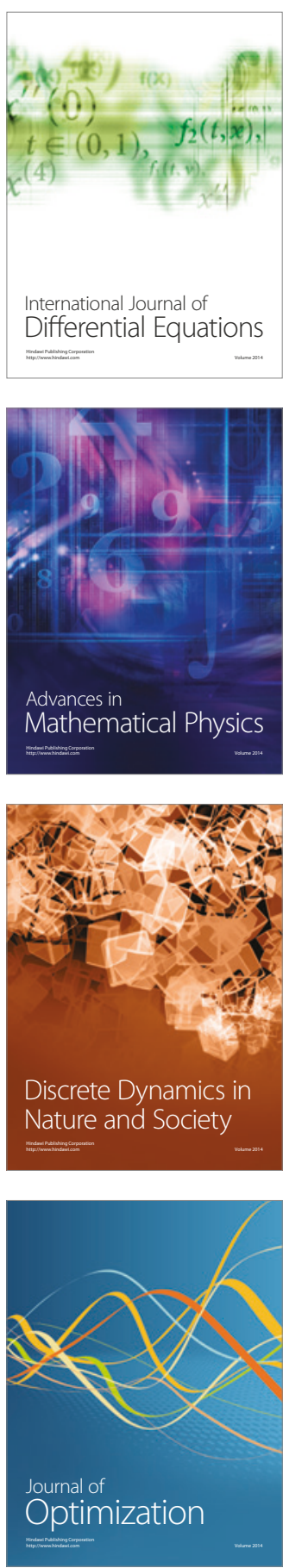